\title{
Strategies to improve sorafenib efficacy during image-guided treatment of hepatocellular carcinoma
}

\author{
Aydin Eresen ${ }^{1}$, Zhuoli Zhang ${ }^{1,2,3}$, Vahid Yaghmai ${ }^{1,3}$ \\ ${ }^{1}$ Department of Radiological Sciences, University of California Irvine, Irvine, CA, USA; ${ }^{2}$ Department of Biomedical Engineering, University of \\ California Irvine, Irvine, CA, USA; ${ }^{3}$ Chao Family Comprehensive Cancer Center, University of California Irvine, Irvine, CA, USA \\ Contributions: (I) Conception and design: All authors; (II) Administrative support: A Eresen, Z Zhang; (III) Provision of study materials or patients: \\ All authors; (IV) Collection and assembly of data: All authors; (V) Data analysis and interpretation: All authors; (VI) Manuscript writing: All authors; \\ (VII) Final approval of manuscript: All authors. \\ Correspondence to: Vahid Yaghmai, MD. Department of Radiological Sciences, School of Medicine, University of California Irvine, 101 City Drive \\ South, Bldg. 1, Rt. 140, Orange, CA 92868, USA. Email: vyaghmai@hs.uci.edu.
}

\begin{abstract}
Hepatocellular carcinoma (HCC) is the third most frequent source of deaths associated with cancer after lung cancer in the world despite recent innovative treatment techniques. Liver transplantation, hepatic resection, and percutaneous ablation techniques hold great promise as potentially curative treatments for patients at early stages. Nevertheless, most of the patients are not suitable for these curative treatments due to their advanced disease stages at the time of diagnosis. Food and Drug Administration (FDA) approved tyrosine kinase inhibitor, sorafenib is a standard therapy for advanced-stage HCC patients which extends overall survival for several months. However, its therapeutic efficacy is restricted by adverse events and drug resistance which limits the number of patients benefiting from this systemic chemotherapeutic drug. During the last decade, novel approaches including but not limited to immunotherapies, ablation methods, and chemotherapeutic drugs were proposed to enhance sensitivity to sorafenib, improve therapeutic efficacy, and prohibit adverse events through novel delivery routes, utilization of nanoparticle carriers, and combination with other therapeutic agents. However, studies are still being conducted to optimize the efficiency of sorafenib and reduce its adverse events. In this review paper, we examine research studies evaluating novel delivery methods to reduce drug-related cytotoxicity to improve patient tolerance to sorafenib and its therapeutic efficacy in patients with HCC. Moreover, therapeutic approaches with the synergistic potential to combine with sorafenib are briefly summarized.
\end{abstract}

Keywords: Hepatocellular carcinoma (HCC); image-guided treatment; nanoparticles; sorafenib

Submitted Jul 21, 2021. Accepted for publication Oct 22, 2021.

doi: $10.21037 /$ atm-21-3768

View this article at: https://dx.doi.org/10.21037/atm-21-3768

\section{Introduction}

Liver and bile duct cancers are expected to lead to 830,180 deaths worldwide in the year 2020. Although the overall survival (OS) of patients with liver cancer has improved by up to $18 \%$ in the last four decades, incidence rate has tripled, and mortality rate has doubled during the same time $(1,2)$. Patients at the early stages of hepatocellular carcinoma (HCC) have the potential for curative treatments such as liver transplantation, hepatic resection, and percutaneous ablation techniques (3). However, only a minority of patients with HCC are suitable candidates for surgical operations because of their advanced disease status at the time of diagnosis (4). The traditional cancer treatment options e.g., radiotherapy and chemotherapy, are still the most frequently performed approaches for patients with HCC. However, adverse events and tumor resistance restrict the potential benefits of these treatments.

Among HCC patients, inflammation and recurrent hepatic regeneration are the most commonly observed etiological features associated with increased cytokines 


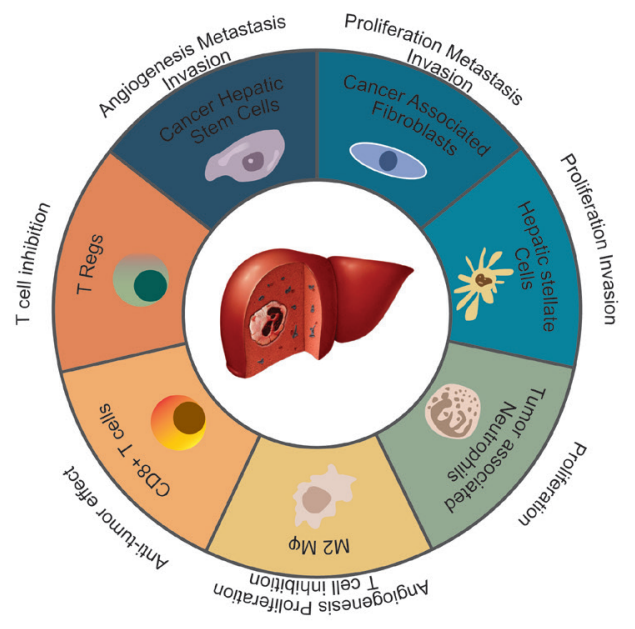

Figure 1 A demonstration of the cell types and their associated effects during the progression of liver cancer. $M \varphi$, macrophages; $\mathrm{T}$ Regs, regulatory $\mathrm{T}$ cells.

and growth factors forming a cancerous environment (5). Figure 1 summarizes cell types and their effects on HCC disease. Although various multikinase inhibitors have been investigated for systemic treatment of HCC, only a limited number of drugs demonstrated promising results and received U.S. Food and Drug Administration (FDA) approval as first-line treatment for advanced-stage HCC patients (6).

The staging of cancer patients is the primary procedure that determines the selection of treatment options, prognosis, and evaluation of therapeutic response. The stage of HCC is described by the evaluation of tumor characteristics reflected on noninvasive medical images. Based on the recommendation of the European Association for the Study of the Liver (EASL) committee, prognostic estimates should be performed through assessment of tumor stage, liver function impairment severity, general health status of the patients, and therapeutic efficacy. Briefly, survival length of the patients is directly correlated with tumor stage. At present, eight different staging systems are proposed for HCC management (7-14). Among them, Barcelona Clinic Liver Cancer (BCLC) staging system, which integrates the knowledge of tumor stage, functional status of the liver, physical status, and cancer-related symptoms, is assumed to be more descriptive than other systems in terms of prognosis $(8,15)$.

In clinical practice, systemic pharmacotherapy is the therapeutic option for advanced-stage HCC. Sorafenib, a small molecule multiple-target tyrosine kinase inhibitor, received FDA approval after the successful completion of a groundbreaking clinical trial that demonstrated prolonged median survival in patients with advanced HCC (16). Sorafenib targets Raf-1, B-Raf, and kinase activity in the Ras/Raf/MEK/ERK signaling pathways to facilitate suppression of tumor cell proliferation and tumor angiogenesis. Recently, atezolizumab plus bevacizumab that received FDA approval as a first-line treatment for HCC patients showed better survival outcomes and progressionfree survival than sorafenib (17); however, the higher cost of this treatment compared to sorafenib is a restricting factor (18). Locoregional therapies, such as thermal ablation, have shown good outcomes for small lesions with limited benefits for tumors $>3 \mathrm{~cm}$ or multifocal disease $(19,20)$. Catheter-directed intrahepatic arterial (IHA) therapies showed statistically significant improvements in OS compared to supportive care (4), yet, these survival benefits remain relatively modest. Therefore, sorafenib is still a key element of the treatment strategy for advanced-stage HCC. However, only $30 \%$ of the patients with advanced HCC will likely benefit from sorafenib as they develop drug resistance within 6 months of the treatment (21). Thus, efficient strategies are required to advance anticancer treatment responses in these patients.

In the past decade, various innovative materials were proposed to carry small molecules and control release rates of sorafenib, providing an alternative drug delivery approach (22). Stimuli-responsive polymers (23), microparticles (24), and nanoparticles (25), and porous materials (26) are used as drug carriers that allow controlled and triggered release of small molecules to improve the amount of drug delivered to targeted regions. Several researchers have investigated different approaches to boost the efficacy of sorafenib by developing new formulations and administration methods $(27,28)$. Xiao et al. targeted the proposed utilization of sorafenib and gadolinium (Gd) co-loaded liposomes (SF/Gd-liposomes) to overcome the limited solubility of sorafenib and to monitor in-vivo treatment efficiency using MRI (27). Zhao et al. examined the potential benefits of regulated delivery of sorafenib and ursolic acid for enhancing therapeutic efficiency while reducing toxicity and dealing with drug resistance using nanoparticle material (28). These studies demonstrated the potential pathway for the improvement of the anticancer activity of sorafenib in clinical settings. For this review, we have searched PubMed and Web of Science databases to determine relevant articles published in English until March 1, 2021, and summarized recent investigations for image- 
guided sorafenib delivery systems in patients with HCC. We present the following article in accordance with the Narrative Review reporting checklist (available at https:// dx.doi.org/10.21037/atm-21-3768).

\section{Sorafenib}

Sorafenib was the first and only therapeutic approach for advanced HCC until acceptance of Lenvatinib as frontline treatment in 2018. Being an orally administered multikinase inhibitor, it inhibits various cell surfaces e.g., vascular endothelial growth factor receptors (VEGF-1, VEGF-2, and VEGF-3), platelet-derived growth factor receptor $\beta$ (PDGFR- $\beta$ ), RET, c-KIT, and FMS-like tyrosine kinase-3 (29). Moreover, sorafenib focuses on serine-threonine kinases Raf- 1 and B-Raf which regulate genes promoting cell proliferation and angiogenesis (30-32) and interacts with tumor cell mechanism at different levels such as blocking Raf/MEK/ERK pathway, reducing angiogenesis through blocking vascular endothelial growth factor/platelet-derived growth factor receptors. Previous preclinical studies demonstrated suppressive action in liver cancer cell lines, decreased tumor angiogenesis and tumor signaling, and intensified tumor-cell apoptosis in a mouse xenograft HCC model (16).

Sorafenib had received FDA approval following successful results of phase III randomized placebocontrolled clinical trials conducted in America and Europe (16), and Asia (33). The median OS in SHARP trial was 10.7 and 7.9 months for patients who received sorafenib or placebo, respectively (16). Moreover, median OS was 6.5 and 4.2 months for sorafenib and placebo groups during a phase III trial conducted in Asia (33). Sorafenib was well-tolerated among the patients that enrolled in either SHARP or Asia-Pacific clinical trials, yet several adverse effects have been observed. The severe adverse event ratio for SHARP trial was recorded as $52 \%$ for treatment and $54 \%$ for placebo groups. However, the incidence of grade 3 drug-related adverse events including hand-foot skin reaction (HFSR) (8\%) and diarrhea (8\%) was higher in the sorafenib group compared to the placebo group. The rate of the patients who left the trial was similar in both groups (38\% in the sorafenib group $v s .37 \%$ in the placebo group); $11 \%$ of the patients that received sorafenib permanently discontinued treatment due to higher rate of toxicity. HFSR and diarrhea were the most common cause for dosage reduction but both adverse events were well managed.

The most common adverse events associated with sorafenib therapy that can impact the quality of the patient life are HFSR, rash, diarrhea, fatigue, dyspepsia, and cardiovascular events. By keeping hands and feet moisturized, removing hyperkeratosis, wearing comfortable shoes, and preventing interaction with hot water, the discomfort related to HFSR can be treated and the life quality of the patients is easily improved (34-39). The majority of the rashes associated with the sorafenib treatment can frequently occur during the first two cycles and mostly can be relieved by using mild, perfume-free soaps, wearing natural fabric clothes, evading hot water as well as topical corticosteroids or antihistamines (40-42). During sorafenib treatment, gastrointestinal disturbances including diarrhea, nausea, vomiting, and anorexia are commonly observed among the patients; however, they don't induce dose reduction unless grade $3 / 4$ severity. Diarrhea can be managed by symptomatic approaches e.g., dietary adjustments, electrolyte compensation, or administering pharmaceutical approaches, e.g., loperamide (42-44). Fatigue generally occurs at the beginning of the sorafenib therapy and disappears following approximately 5-6 months of the administration. Due to the higher potential of association with other underlying causes, the condition of the patients should be carefully evaluated, and symptoms of fatigue should be targeted (42). Hypertension may occur associated with cytotoxicity of sorafenib at the early stages of the therapy and may decrease later (45-47). Antihypertensive agents e.g., angiotensinconverting enzyme (ACE) inhibitors and b-blockers can be administered to control hypertension among the HCC patients $(48,49)$.

Despite the proven efficacy of the sorafenib treatment for HCC patients, drug resistance to sorafenib destructs the usage in clinical practice. Sorafenib resistance generally occurs among the patients following 4-6 months of successful clinical improvement. The complexity of the underlying mechanism for sorafenib therapy restricts the determination of the causes for developing drug resistance. According to the previous research, the resistance to the sorafenib drug among HCC patients can be classified as primary/intrinsic or acquired/secondary resistance. As genetic heterogeneity of HCC cells indicates the potentially intrinsic resistance, resistance occurring following longterm exposure to sorafenib treatment associated with EGFR/c-Jun/AKT activation (50-55), autophagy (56-58), hypoxic environment (59-61), epithelial-mesenchymal transition (62-64), and cancer stem cells (65-67). Due to the variation of the mechanism inhibiting drug 

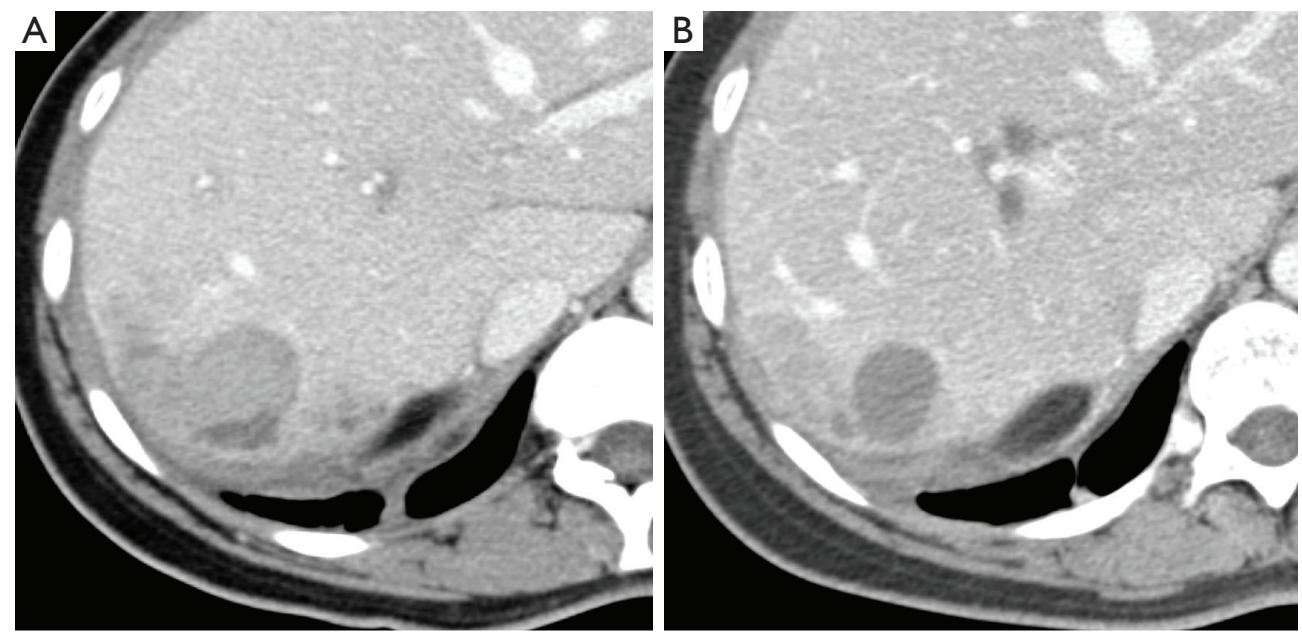

Figure 2 A 36-year-old patient with hepatocellular carcinoma in the right hepatic lobe. (A) CT before administration of sorafenib shows lobulated partially enhancing mass; (B) 4 months after the start of sorafenib, a portion of the mass shows no enhancement, and the mass has decreased in size.

resistance, different strategies have still been investigated in ongoing clinical trials. Due to the complexity of the mechanism, comprehensive investigation is still required for proposing an effective approach for preventing drug resistance. Two recent studies have focused on the symptoms of sorafenib drug resistance in HCC patients and discussed the suggestions to inhibit factors causing resistance and improving the efficacy of the treatment $(68,69)$. In this study, we have summarized the studies that focus on improving the efficacy of sorafenib through the implementation of novel delivery techniques and combination treatment approaches.

\section{Drug delivery}

Standard approach for delivering most cancer drugs has been through the intravenous route $(70,71)$. However, oral administration has gained great attention due to improved patient compliance, drug tolerability, potential efficacy, and reduced cost compared to the parenteral route (71-73). Nonetheless, due to drug-related cytotoxicity and adverse events, alternative delivery routes for therapeutic agents are needed.

\section{Systemic delivery}

In clinical practice, sorafenib is administered through systemic delivery in the form of tablets $(2 \times 200 \mathrm{mg})$ twice daily for treatment of advanced HCC (74). A representative
CT image for a 36 years old patient demonstrated the successful outcome of sorafenib treatment in Figure 2. The effects of the drug dosage during the treatment were also investigated to control the side effects of this drug (75). The study performed by Tak et al. demonstrated significant improvement in progression-free survival among (patients receiving a decreased dose of sorafenib (400 mg per day) compared to patients receiving $800 \mathrm{mg}$ per day and patients receiving $600 \mathrm{mg}$ per day (75). This suggested that dose reduction of sorafenib can advance OS and improve drug tolerance. Despite improving survival benefits, side effects of sorafenib require close attention to monitoring of cytotoxicity and therapeutic response. Horger et al. suggested indirect monitoring of the advanced stage HCC patients receiving sorafenib with conventional MRI technique (76). The patients were imaged using MRI before, approximately 3 weeks, 5 weeks, and 8 weeks after the treatment. MRI demonstrated significant intensity changes on $\mathrm{T} 1 \mathrm{w}$, and $\mathrm{T} 2 \mathrm{w}$ images related to intralesional necrosis caused by sorafenib. Schraml et al. focused on monitoring changes in tumor cellularity and cell membrane integrity for the assessment of therapeutic response (77). The diffusion-weighted (DW) MRI analysis pointed that the apparent diffusion coefficient reflected changes in the tumor microenvironment related to tumor necrosis. Similarly, Shirota et al. utilized DW-MRI data to compute intravoxel coherent motion parameters to assess sorafenib therapy (78). The study highlighted the sensitivity of the true diffusion coefficient for determining response 
to sorafenib. Furthermore, Hsu et al. suggested dynamic contrast-enhanced (DCE) MRI to monitor vascular changes following a combination of sorafenib and metronomic tegafur/uracil therapy for advanced HCC (79). The analysis of DCE-MRI data acquired on the $14^{\text {th }}$ day of the treatment demonstrated a strong variation for the vascular response reflected with $K^{\text {trans }}$. Although these studies presented potential approaches for assessing therapeutic response, active monitoring of sorafenib by imaging has been shown to help reduce adverse events by tracking drug distribution, thus allowing drug dosage adjustment. Grillone et al. proposed encapsulating sorafenib and superparamagnetic iron oxide nanoparticles (SPIONs) to improve drug delivery to targeted tissue via monitoring by MRI (80). Solid lipid nanoparticles were loaded with sorafenib and paramagnetic materials using a hot homogenization technique. This was achieved by using cetyl palmitate as a lipid matrix with $90 \%$ of sorafenib loading efficiency. The results of an in-vitro study performed on human HCC HepG2 cells demonstrated that sorafenib-loaded solid lipid nanoparticles facilitated tumor growth inhibition while allowing active monitoring of the drug distribution with MRI. Zhang et al. investigated therapeutic efficiency of targeted folatefunctionalized micelles loaded with SPIONs and sorafenib by performing an in vitro study on human HepG2 cells (81). The study showed blocked proliferation and reduced apoptosis of HepG2 cells with significant intensity drop on T2w MRI data, enabling monitoring of targeted micelles.

Similarly, in vivo studies have shown promise. Lin et al. investigated the development and characterization of a nanoparticle formulation consisting of poly(ethylene glycol)-b-poly(lactic-co-glycolic acid) (PEG-PLGA) copolymers and poly(lactic-co-glycolic acid) (PLGA) (82). These investigators aimed to accomplish systemic delivery of encapsulated sorafenib into fibrotic livers of $\mathrm{CCI}_{4}$ induced $\mathrm{C} 3 \mathrm{H} / \mathrm{HeNCrNarl}$ mice. The results emphasized that developed PLGA nanoparticles loaded with sorafenib reduced the level of $\alpha$-smooth muscle actin content and collagen in livers of the mice which suggested mitigation of liver fibrosis. Furthermore, a recent study by Shi et al. proposed an approach for oral delivery of sorafenib by the formation of choline and geranic acid (CAGE) nanocomplexes (83). The preclinical study performed on rats demonstrated that CAGE improved the solubility and blood concentration of sorafenib and enhanced the elimination of half-life and mean absorption time of the drug, which suggested the potential benefits of this new approach. Despite the promising results of the studies to improve sorafenib efficacy during the administration of systemic delivery, there is a great need for comprehensive preclinical studies to demonstrate the safety and efficacy of the approaches.

\section{Local delivery}

The low bioavailability of sorafenib is related to its low aqueous solubility, poor intestinal epithelium permeability, high level of P-glycoprotein efflux, liver cytochrome P450 metabolic enzymes, and pre-systemic metabolism $(83,84)$. Sorafenib's toxicity requires a narrow therapeutic window in which novel delivery approaches are required to reduce the side effects by improving the absorption of sorafenib at targeted regions (82). To overcome these limitations, researchers have proposed local delivery of the cancer drugs by encapsulating them with nanoparticles arranging in size from 5 to $200 \mathrm{~nm}$, depending on load and the utilized material $(85,86)$.

In recent years, utilization of nanoparticles to deliver sorafenib to HCC tumors has been studied extensively. The small size and larger surface of the nanoparticles loaded with sorafenib improved the solubility of this drug. Moreover, adjustments to the characteristics of nanoparticles have allowed the delivery of sorafenib to targeted tumors and improved the cellular response $(87,88)$. By controlling drug release, dosage and intake frequency can be decreased, allowing for reduced cytotoxicity (88). Nanoparticles can be loaded with magnetic particles that allow tracking and monitoring release of the drugs triggered by the acidic microenvironment of tumor tissue (89). Moreover, sorafenib-loaded nanoparticles can be delivered to targeted tumor locations by image guidance, bypassing the barriers that block conventional drugs for the effective treatment of cancers. The activity of nanoparticles for optimized drug delivery is shown in Figure 3.

Craparo et al. proposed liver-targeted polymeric micelles for focused delivery of sorafenib to the liver for treatment of HCC (91). The researchers prepared an average of $101.8 \pm 64.3 \mathrm{~nm}$-sized sorafenib-loaded micelles through a chemical reaction of a,b-poly(N-2-hydroxyethyl) (2-aminoethylcarbamate)-D,L-aspartamide (PHEA-EDA) with polylactic acid (PLA), and subsequent reaction with lactose. The preclinical biodistribution study demonstrated the focused accumulation of sorafenib in mice livers. A preclinical study performed by Chen et al. examined development of poly(lactide-co-glycolide) microspheres 


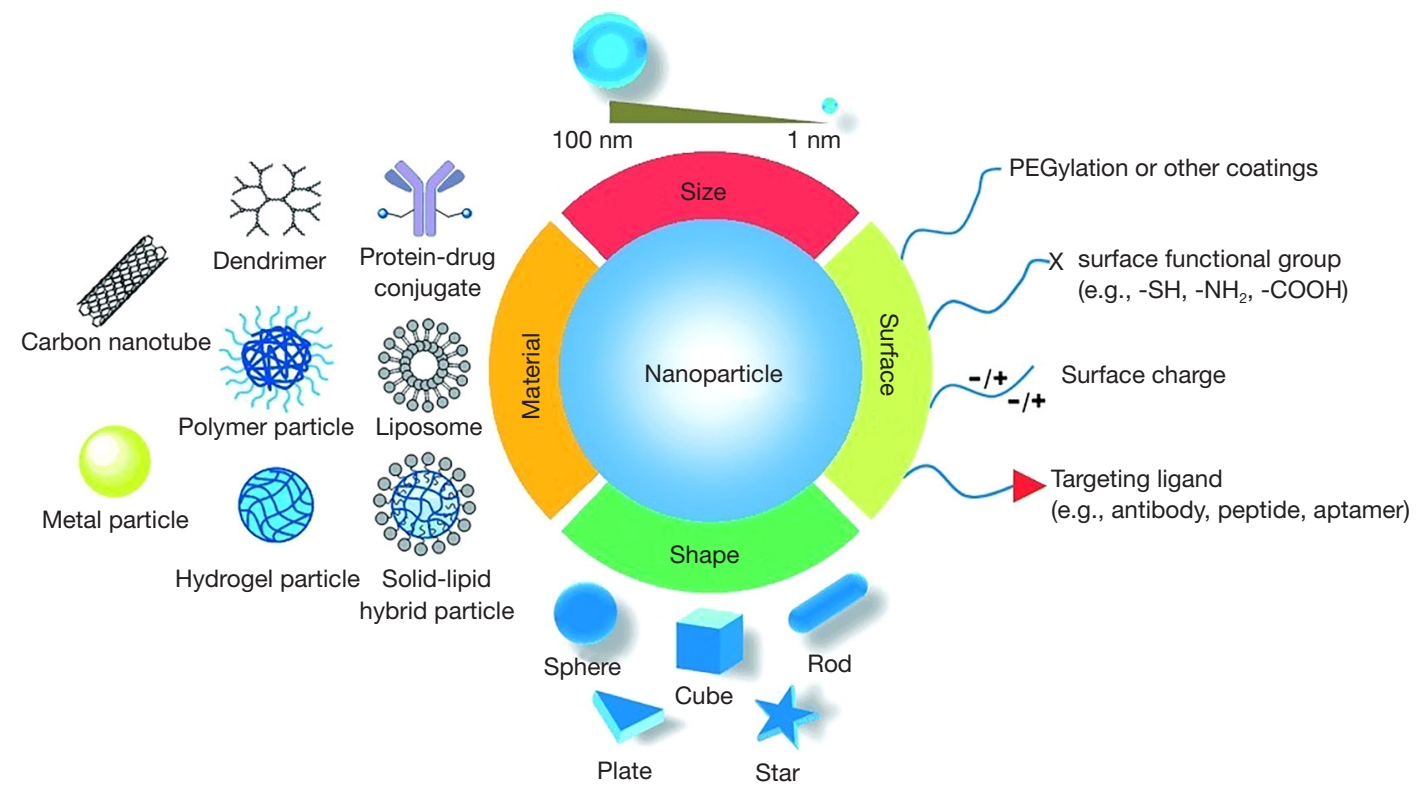

Figure 3 The nanoparticles utilized for drug delivery and their biophysicochemical properties. Reused with permission from "Engineered nanoparticles for drug delivery in cancer therapy" by Sun et al. (90). Copyright () 2021 John Wiley and Sons.

for local delivery of sorafenib while co-encapsulating with iron oxide nanoparticles to allow monitoring of microsphere delivery through MRI (92). The microspheres with a size of 13 microns enabled the release of sorafenib within 72 hours, and the results of the study performed on VX2 rabbits demonstrated the normalized VEGFR-2 activity and decreased microvessel density. The structure of polymer nanoparticles can support localized delivery of the therapeutic drug which may reduce drug intake and improve tolerance. Hence, they are commonly utilized in activities for the treatment of HCC (93). Moreover, Xiao et al. proposed a liposome loaded with sorafenib and gadolinium to improve poor water solubility and monitor the distribution of sorafenib using a non-invasive imaging approach (27). Their study highlighted that sorafenib encapsulated with liposome significantly improved water solubility from 0.21 to $250 \mu \mathrm{g} / \mathrm{mL}$ and lower cell cytotoxicity was observed with slow and sustained release of the drug. Liposomes that were spherical or ellipsoid-shaped with a uniform particle size showed high drug load capacity and demonstrated better antitumor activity compared to oral delivery in H22 tumor-bearing mice. In-vivo studies, gadolinium encapsulated with liposome enabled longer MRI acquisition time with higher signal intensity.

Hepatic arterial supply of HCC allows transarterial chemoembolization for targeted delivery of chemotherapeutic agents and devascularization of the tumors. The embolization of the hepatic arteries that supply tumors facilitates hypoxia and necrosis, yet new blood vessels are also generated (94). Gaba et al. proposed local delivery of tyrosine kinase inhibitor sorafenib via transarterial administration to prevent ampelotherapyinduced angiogenesis (95). The preclinical study that was performed using the rabbit VX2 HCC model resulted in a higher concentration of sorafenib with targeted local delivery compared to drug levels observed via systemic delivery. The histopathological analysis showed mild or moderate non-specific swelling in zone 3 hepatocytes without sign of necrosis.

In a recent study, Ning and Wang investigated the efficacy, feasibility, and safety of intrahepatic delivery of sorafenib by performing a preclinical study using normal liver tissues of the rabbits (96). The study compared local delivery of sorafenib via transcatheter selective hepatic arterial embolization (group I) and hepatic arterial infusion (group II). The results demonstrated that sorafenib peak concentration for group I $(2.46 \pm 0.101 \mu \mathrm{g} / \mathrm{mL})$ was significantly lower than group II $(3.78 \pm 0.180 \mu \mathrm{g} / \mathrm{mL})$ while local necrosis occurred in subjects of group I but not group II. The authors highlighted the feasibility and safety of sorafenib delivery via transcatheter selective hepatic arterial embolization while observing a slow-release 
effect. Furthermore, Zhang et al. demonstrated therapeutic efficiency of the hepatic arterial delivery of sorafenib in the VX2 rabbit HCC model (97). The preclinical study resulted in decreased tumor growth and intrahepatic metastasis without hepatic or renal toxicity, suggesting the safe and effective use of hepatic arterial administration of sorafenib for HCC. In a recent study, Li et al. proposed encapsulation of sorafenib and catalase using poly(lacticco-glycolic acid) (PLGA) microspheres to inhibit tumor hypoxia and hypoxia-related tumor angiogenesis associated with transcatheter arterial chemoembolization (98). The in vitro/in vivo study demonstrated that PLGA microspheres loaded with sorafenib and catalase advanced efficacy of hepatic artery embolization, regulated tumor hypoxia, and immunosuppressive tumor microenvironment by facilitating rapid necrosis of the tumors. On the other hand, Park et al. investigated potential approaches for improving drugeluting beads developed for transcatheter hepatic intraarterial injection delivery to HCC (99). By preparing an acidic $\mathrm{pH}$-triggered drug-eluting nanocomposite material combined with SPIONs and $\mathrm{pH}$-responsive synthetic peptides with lipid tails, they enabled sorafenib delivery while monitoring drug release behavior using MRI. The preclinical study results performed on an orthotopic rat HCC model demonstrated that transcatheter hepatic intra-arterial delivery of sorafenib-loaded nanocomposites had great promise for inhibition of tumor growth while suggesting the proposed platform to deliver sorafenib drug for the treatment of unresectable HCC. Despite the potential mechanism of local delivery of the drugs, the need for more frequent hospital visits will be a limiting factor for this approach. Therefore, minimally invasive strategies are required to advance the feasibility of the local delivery of drugs.

\section{Combination therapy with sorafenib}

Despite being the standard therapy for advanced-stage HCC, the majority of patients cannot benefit from sorafenib due to the genetic heterogeneity of HCC (100). Therefore, sorafenib can only extend the OS of the patients for several months (101). The shortcomings of sorafenib drug therapy have led to research of novel and efficient approaches for the treatment of HCC. With the potential synergistic role of combination therapy, researchers have actively investigated the supportive treatment techniques to apply in conjunction with sorafenib for improved outcomes. In earlier studies, researchers applied sorafenib in a combination with transcatheter arterial chemoembolization (TACE), standard palliative therapy for HCC, to assess for the improved outcomes through the synergy of two standard approaches (102-104). However, studies completed at different centers resulted in different outcomes potentially caused by regional differences and genetic heterogeneity $(102,105)$. Phase III SHARP trial demonstrated that combination of sorafenib and TACE did not improve the progression-free survival (106) while combination therapy significantly improved OS of the patients compared with monotherapies in retrospective studies (107-111). Ricke et al. evaluated the potential impact of the combination of sorafenib and selective yttrium-90 transarterial radioembolization and showed that combination treatment did not improve OS of the patients compared to sorafenib only therapy (112). However, a retrospective study that included 4,763 patients diagnosed with HCC showed improved OS of the patients that underwent radiotherapy within sorafenib use compared to patients that only received sorafenib therapy (113). Moreover, a recent clinical trial demonstrated that a combination of sorafenib, hepatic arterial infusion chemotherapy of oxaliplatin, fluorouracil, and leucovorin, and FOLFOX enhances OS of advancedstage HCC patients compared to individuals who received sorafenib monotherapy (114). Moreover, two ongoing clinical trials (NCT03211416 and NCT02562755) focusing on the combination of sorafenib and pembrolizumab, and sorafenib plus pexa-Vec therapies are under investigation for potential first-line therapy for HCC.

With the recent advancement of knowledge and technology, immune checkpoint inhibitors have attracted research to develop novel approaches for immunotherapy of HCC. Recent FDA approval of nivolumab and pembrolizumab followed the satisfactory results of phase I/II clinical trials and comparable benefits of phase III trials with sorafenib monotherapy and placebo $(115,116)$. Despite the promising results of some clinical trials, these approaches may be influenced by immunosuppression lead by VEGF/VEGFR pathway (117-119). The preclinical study performed by Chen et al. demonstrated that antiPD-1 monotherapy facilitates anti-tumor activities that were further enhanced through a combination of sorafenib and AMD3100 (120). Zhou et al. investigated therapeutic efficiency and safety of the combination of dendritic and cytokine killer cells with sorafenib for the treatment of individuals with HCC (121). The clinical study resulted in significantly improved OS in patients who received combination therapy (18.6 months) compared 
to patients treated with sorafenib only (13.8 months). Furthermore, currently ongoing phase II/III clinical trials (NCT04152356, NCT04518852, NCT01658878, and NCT04229355) are evaluating potential outcomes of the combination of immunotherapy with systemic therapies for patients with HCC. The complexity of the disease mechanism utmost challenges combinatorial therapies despite synergistic expectations to utilize therapies that target multiple pathways to destruct the tumor microenvironment. Therefore, a great need for comprehensive studies is present to identify the most promising combinatorial agents for the treatment of HCC.

\section{Future perspectives and summary}

Despite promising benefits for the treatment of HCC patients, drug resistance and adverse events cause postponing or stopping sorafenib administration for the majority of the patients. In the light of the recent advancement in immunotherapy and chemotherapeutic drugs, the combination of sorafenib with other treatment approaches targeting various intracellular signaling pathways may overcome the monotherapy failure by reducing the acquired resistance as well as improving antitumor activity $(54,122,123)$. Zhai et al. demonstrated that inhibition of Akt pathway might be a synergistic approach to reverse acquired resistance to sorafenib through adapting autophagy for death-promoting mechanism during sorafenib treatment (54). Besides, Ardelt et al. have investigated the potential benefits for a combination of cyclin-dependent kinase $5(\mathrm{Cdk} 5)$ and sorafenib (123). This combinational treatment approach demonstrated an impaired HCC progression in vitro and in vivo studies while curbing tumor cell proliferation and migration. Due to the complexity of molecular mechanism is of utmost importance for planning effective and safe combinational drug candidates for the treatment of patients with advanced HCC patients.

Only $10-15 \%$ of the patients with HCC are suitable for promising curative treatments e.g., surgical resection and liver transplantation. Sorafenib remains the first-line therapy for advanced-stage HCC patients. Despite the proven improvement of OS, the adverse events and drug resistance limit the number of patients that can benefit from sorafenib. With the advancement of technology, novel drug delivery techniques (e.g., local delivery with the use of carriers) are being proposed to reduce drug-related cytotoxicity. Moreover, sorafenib plus other therapeutic agents have been investigated to improve sensitivity to sorafenib and inhibit drug-related side effects while improving the efficacy of treatment. Recent studies have examined potential combinatorial agents that interact with the mechanism of sorafenib and advance the clinical outcome synergistically. The studies have demonstrated the promising benefits of a combination of sorafenib and immunotherapy to improve the treatment efficacy and inhibit adverse events. However, more studies in patients with advanced HCC are required to evaluate the clinical benefits for localized delivery of combinatorial therapies that include sorafenib.

\section{Acknowledgments}

Funding: This study was supported by the National Cancer Institute (grants R01CA196967, R01CA209886, and R01CA241532), and Society of Interventional Radiology Foundation pilot grant (PR-0000000012).

\section{Footnote}

Reporting Checklist: The authors have completed the Narrative Review reporting checklist. Available at https:// dx.doi.org/10.21037/atm-21-3768

Conflicts of Interest: All authors have completed the ICMJE uniform disclosure form (available at https://dx.doi. org/10.21037/atm-21-3768). AE reports a grant from Society of Interventional Radiology (PR-0000000012). $\mathrm{ZZ}$ reports grant from National Institute of Health (R01CA196967, R01CA209886, and R01CA241532). VY reports a grant from National Institute of Health (grant R01CA241532).

Ethical Statement: The authors are accountable for all aspects of the work in ensuring that questions related to the accuracy or integrity of any part of the work are appropriately investigated and resolved.

Open Access Statement: This is an Open Access article distributed in accordance with the Creative Commons Attribution-NonCommercial-NoDerivs 4.0 International License (CC BY-NC-ND 4.0), which permits the noncommercial replication and distribution of the article with the strict proviso that no changes or edits are made and the original work is properly cited (including links to both the formal publication through the relevant DOI and the license). See: https://creativecommons.org/licenses/by-nc-nd/4.0/. 


\section{References}

1. American Cancer Society. Key Statistics About Liver Cancer 2020. Available online: https://www.cancer.org/ cancer/liver-cancer/about/what-is-key-statistics.html

2. American Cancer Society. Global Cancer Facts \& Figures 2019. Available online: https://www.cancer.org/content/ dam/cancer-org/research/cancer-facts-and-statistics/ annual-cancer-facts-and-figures/2019/cancer-facts-andfigures-2019.pdf

3. Lencioni R, de Baere T, Soulen MC, et al. Lipiodol transarterial chemoembolization for hepatocellular carcinoma: A systematic review of efficacy and safety data. Hepatology 2016;64:106-16.

4. Llovet JM, Real MI, Montaña X, et al. Arterial embolisation or chemoembolisation versus symptomatic treatment in patients with unresectable hepatocellular carcinoma: a randomised controlled trial. Lancet 2002;359:1734-9.

5. Mantovani A, Allavena P, Sica A, et al. Cancer-related inflammation. Nature 2008;454:436-44.

6. Sun Y, Ma W, Yang Y, et al. Cancer nanotechnology: Enhancing tumor cell response to chemotherapy for hepatocellular carcinoma therapy. Asian J Pharm Sci 2019;14:581-94.

7. Okuda K, Ohtsuki T, Obata H, et al. Natural history of hepatocellular carcinoma and prognosis in relation to treatment. Study of 850 patients. Cancer 1985;56:918-28.

8. Llovet JM, Brú C, Bruix J. Prognosis of hepatocellular carcinoma: the BCLC staging classification. Semin Liver Dis 1999; 19:329-38.

9. Chevret S, Trinchet JC, Mathieu D, et al. A new prognostic classification for predicting survival in patients with hepatocellular carcinoma. Groupe d'Etude et de Traitement du Carcinome Hépatocellulaire. J Hepatol 1999;31:133-41.

10. Prospective validation of the CLIP score: a new prognostic system for patients with cirrhosis and hepatocellular carcinoma. The Cancer of the Liver Italian Program (CLIP) Investigators. Hepatology 2000;31:840-5.

11. Leung TW, Tang AM, Zee B, et al. Construction of the Chinese University Prognostic Index for hepatocellular carcinoma and comparison with the TNM staging system, the Okuda staging system, and the Cancer of the Liver Italian Program staging system: a study based on 926 patients. Cancer 2002;94:1760-9.

12. Vauthey JN, Lauwers GY, Esnaola NF, et al. Simplified staging for hepatocellular carcinoma. J Clin Oncol
2002;20:1527-36.

13. Kudo M, Chung H, Osaki Y. Prognostic staging system for hepatocellular carcinoma (CLIP score): its value and limitations, and a proposal for a new staging system, the Japan Integrated Staging Score (JIS score). J Gastroenterol 2003;38:207-15.

14. Villa E, Colantoni A, Cammà C, et al. Estrogen receptor classification for hepatocellular carcinoma: comparison with clinical staging systems. J Clin Oncol 2003;21:441-6.

15. Subramaniam S, Kelley RK, Venook AP. A review of hepatocellular carcinoma (HCC) staging systems. Chin Clin Oncol 2013;2:33.

16. Llovet JM, Ricci S, Mazzaferro V, et al. Sorafenib in advanced hepatocellular carcinoma. N Engl J Med 2008;359:378-90.

17. Finn RS, Qin S, Ikeda M, et al. Atezolizumab plus Bevacizumab in Unresectable Hepatocellular Carcinoma. N Engl J Med 2020;382:1894-905.

18. Wang W, Wang J, Zhang X, et al. Atezolizumab plus bevacizumab for unresectable or metastatic hepatocellular carcinoma: A cost-effectiveness analysis. J Clin Oncol 2021;39:295.

19. Ahmed M, Goldberg SN. Thermal ablation therapy for hepatocellular carcinoma. J Vasc Interv Radiol 2002;13:S231-44.

20. Clark TW, Soulen MC. Chemical ablation of hepatocellular carcinoma. J Vasc Interv Radiol 2002;13:S245-52.

21. Abdel-Rahman O, Abdel-Wahab M, Shaker M, et al. Sorafenib versus capecitabine in the management of advanced hepatocellular carcinoma. Med Oncol 2013;30:655.

22. Wang Y, Kohane DS. External triggering and triggered targeting strategies for drug delivery. Nat Rev Mater 2017;2:17020.

23. Fu X, Hosta-Rigau L, Chandrawati R, et al. Multi-StimuliResponsive Polymer Particles, Films, and Hydrogels for Drug Delivery. Chem 2018;4:2084-107.

24. Kumar A, Montemagno C, Choi HJ. Smart Microparticles with a $\mathrm{pH}$-responsive Macropore for Targeted Oral Drug Delivery. Sci Rep 2017;7:3059.

25. Yoo JW, Doshi N, Mitragotri S. Adaptive micro and nanoparticles: temporal control over carrier properties to facilitate drug delivery. Adv Drug Deliv Rev 2011;63:1247-56.

26. Qiu L, Zhao Y, Li B, et al. Triple-stimuli (protease/redox/ $\mathrm{pH})$ sensitive porous silica nanocarriers for drug delivery. Sens Actuators B Chem 2017;240:1066-74. 
27. Xiao Y, Liu Y, Yang S, et al. Sorafenib and gadolinium coloaded liposomes for drug delivery and MRI-guided HCC treatment. Colloids Surf B Biointerfaces 2016;141:83-92.

28. Zhao R, Li T, Zheng G, et al. Simultaneous inhibition of growth and metastasis of hepatocellular carcinoma by co-delivery of ursolic acid and sorafenib using lactobionic acid modified and $\mathrm{pH}$-sensitive chitosanconjugated mesoporous silica nanocomplex.

Biomaterials 2017;143:1-16.

29. Raza A, Sood GK. Hepatocellular carcinoma review: current treatment, and evidence-based medicine. World J Gastroenterol 2014;20:4115-27.

30. Wilhelm S, Carter C, Lynch M, et al. Discovery and development of sorafenib: a multikinase inhibitor for treating cancer. Nat Rev Drug Discov 2006;5:835-44.

31. Marshall CJ. MAP kinase kinase kinase, MAP kinase kinase and MAP kinase. Curr Opin Genet Dev 1994;4:82-9.

32. Wilhelm SM, Carter C, Tang L, et al. BAY 43-9006 exhibits broad spectrum oral antitumor activity and targets the RAF/MEK/ERK pathway and receptor tyrosine kinases involved in tumor progression and angiogenesis. Cancer Res 2004;64:7099-109.

33. Cheng AL, Kang YK, Chen Z, et al. Efficacy and safety of sorafenib in patients in the Asia-Pacific region with advanced hepatocellular carcinoma: a phase III randomised, double-blind, placebo-controlled trial. Lancet Oncol 2009;10:25-34.

34. Ren Z, Zhu K, Kang H, et al. Randomized controlled trial of the prophylactic effect of urea-based cream on sorafenib-associated hand-foot skin reactions in patients with advanced hepatocellular carcinoma. J Clin Oncol 2015;33:894-900.

35. Anderson R, Jatoi A, Robert C, et al. Search for evidencebased approaches for the prevention and palliation of hand-foot skin reaction (HFSR) caused by the multikinase inhibitors (MKIs). Oncologist 2009;14:291-302.

36. Autier J, Escudier B, Wechsler J, et al. Prospective study of the cutaneous adverse effects of sorafenib, a novel multikinase inhibitor. Arch Dermatol 2008;144:886-92.

37. Frenette CT, Frederick RT, Gish RG. Clinical management and case reports for the treatment of hepatocellular carcinoma with sorafenib. J Clin Gastroenterol 2011;45:733-7.

38. Lacouture ME, Reilly LM, Gerami P, et al. Hand foot skin reaction in cancer patients treated with the multikinase inhibitors sorafenib and sunitinib. Ann Oncol 2008;19:1955-61.
39. Manchen E, Robert C, Porta C. Management of tyrosine kinase inhibitor-induced hand-foot skin reaction: viewpoints from the medical oncologist, dermatologist, and oncology nurse. J Support Oncol 2011;9:13-23.

40. Wood LS. Managing the side effects of sorafenib and sunitinib. ommunity Oncol 2006;3:558-62.

41. Pérez-Soler R, Delord JP, Halpern A, et al. HER1/ EGFR inhibitor-associated rash: future directions for management and investigation outcomes from the HER1/ EGFR inhibitor rash management forum. Oncologist 2005;10:345-56.

42. Brose MS, Frenette CT, Keefe SM, et al. Management of sorafenib-related adverse events: a clinician's perspective. Semin Oncol 2014;41 Suppl 2:S1-S16.

43. Walko CM, Grande C. Management of common adverse events in patients treated with sorafenib: nurse and pharmacist perspective. Semin Oncol 2014;41 Suppl 2:S17-28.

44. Li Y, Gao ZH, Qu XJ. The adverse effects of sorafenib in patients with advanced cancers. Basic Clin Pharmacol Toxicol 2015;116:216-21.

45. Roodhart JM, Langenberg MH, Witteveen E, et al. The molecular basis of class side effects due to treatment with inhibitors of the VEGF/VEGFR pathway. Curr Clin Pharmacol 2008;3:132-43.

46. Shin SY, Lee YJ. Correlation of skin toxicity and hypertension with clinical benefit in advanced hepatocellular carcinoma patients treated with sorafenib. Int J Clin Pharmacol Ther 2013;51:837-46.

47. Jain L, Sissung TM, Danesi R, et al. Hypertension and hand-foot skin reactions related to VEGFR2 genotype and improved clinical outcome following bevacizumab and sorafenib. J Exp Clin Cancer Res 2010;29:95.

48. Maitland ML, Bakris GL, Black HR, et al. Initial assessment, surveillance, and management of blood pressure in patients receiving vascular endothelial growth factor signaling pathway inhibitors. J Natl Cancer Inst 2010;102:596-604.

49. Larochelle P, Kollmannsberger C, Feldman RD, et al. Hypertension management in patients with renal cell cancer treated with anti-angiogenic agents. Curr Oncol 2012;19:202-8.

50. Ezzoukhry Z, Louandre C, Trécherel E, et al. EGFR activation is a potential determinant of primary resistance of hepatocellular carcinoma cells to sorafenib. Int J Cancer 2012;131:2961-9.

51. Blivet-Van Eggelpoël MJ, Chettouh H, Fartoux L, et al. Epidermal growth factor receptor and HER-3 restrict cell 
response to sorafenib in hepatocellular carcinoma cells. J Hepatol 2012;57:108-15.

52. Chen W, Xiao W, Zhang K, et al. Activation of c-Jun predicts a poor response to sorafenib in hepatocellular carcinoma: Preliminary Clinical Evidence. Sci Rep 2016;6:22976.

53. Haga Y, Kanda T, Nakamura M, et al. Overexpression of c-Jun contributes to sorafenib resistance in human hepatoma cell lines. PLoS One 2017;12:e0174153.

54. Zhai B, Hu F, Jiang X, et al. Inhibition of Akt reverses the acquired resistance to sorafenib by switching protective autophagy to autophagic cell death in hepatocellular carcinoma. Mol Cancer Ther 2014;13:1589-98.

55. Chen KF, Chen HL, Tai WT, et al. Activation of phosphatidylinositol 3-kinase/Akt signaling pathway mediates acquired resistance to sorafenib in hepatocellular carcinoma cells. J Pharmacol Exp Ther 2011;337:155-61.

56. Luo T, Fu J, Xu A, et al. PSMD10/gankyrin induces autophagy to promote tumor progression through cytoplasmic interaction with ATG7 and nuclear transactivation of ATG7 expression. Autophagy 2016;12:1355-71.

57. Thorburn A. Autophagy and its effects: making sense of double-edged swords. PLoS Biol 2014;12:e1001967.

58. Fischer TD, Wang JH, Vlada A, et al. Role of autophagy in differential sensitivity of hepatocarcinoma cells to sorafenib. World J Hepatol 2014;6:752-8.

59. Kim JY, Lee JY. Targeting Tumor Adaption to Chronic Hypoxia: Implications for Drug Resistance, and How It Can Be Overcome. Int J Mol Sci 2017;18:1854.

60. Liang Y, Zheng T, Song R, et al. Hypoxia-mediated sorafenib resistance can be overcome by EF24 through Von Hippel-Lindau tumor suppressor-dependent HIF$1 \alpha$ inhibition in hepatocellular carcinoma. Hepatology 2013;57:1847-57.

61. Zhao D, Zhai B, He C, et al. Upregulation of HIF-2 $\alpha$ induced by sorafenib contributes to the resistance by activating the TGF- $\alpha$ /EGFR pathway in hepatocellular carcinoma cells. Cell Signal 2014;26:1030-9.

62. Shang Y, Cai X, Fan D. Roles of epithelial-mesenchymal transition in cancer drug resistance. Curr Cancer Drug Targets 2013;13:915-29.

63. Huang XY, Ke AW, Shi GM, et al. $\alpha \mathrm{B}$-crystallin complexes with 14-3-3 $\zeta$ to induce epithelial-mesenchymal transition and resistance to sorafenib in hepatocellular carcinoma. Hepatology 2013;57:2235-47.

64. van Malenstein H, Dekervel J, Verslype C, et al. Longterm exposure to sorafenib of liver cancer cells induces resistance with epithelial-to-mesenchymal transition, increased invasion and risk of rebound growth. Cancer Lett 2013;329:74-83.

65. Park CY, Tseng D, Weissman IL. Cancer stem celldirected therapies: recent data from the laboratory and clinic. Mol Ther 2009; 17:219-30.

66. Tovar V, Cornella H, Moeini A, et al. Tumour initiating cells and IGF/FGF signalling contribute to sorafenib resistance in hepatocellular carcinoma. Gut 2017;66:530-40.

67. Ma MKF, Lau EYT, Leung DHW, et al. Stearoyl-CoA desaturase regulates sorafenib resistance via modulation of ER stress-induced differentiation. J Hepatol 2017;67:979-90.

68. Tang W, Chen Z, Zhang W, et al. The mechanisms of sorafenib resistance in hepatocellular carcinoma: theoretical basis and therapeutic aspects. Signal Transduct Target Ther 2020;5:87.

69. Zeng Z, Lu Q, Liu Y, et al. Effect of the Hypoxia Inducible Factor on Sorafenib Resistance of Hepatocellular Carcinoma. Front Oncol 2021;11:641522.

70. Ruddy K, Mayer E, Partridge A. Patient adherence and persistence with oral anticancer treatment. CA Cancer J Clin 2009;59:56-66.

71. O'Neill VJ, Twelves CJ. Oral cancer treatment: developments in chemotherapy and beyond. Br J Cancer 2002;87:933-7.

72. Jeanneret LA, Schneider MP, Troxler S, et al. Therapeutic adherence to oral cancer therapy and interdisciplinary management. Rev Med Suisse 2011;7:1154-8, 1160.

73. Partridge AH, Avorn J, Wang PS, et al. Adherence to therapy with oral antineoplastic agents. J Natl Cancer Inst 2002;94:652-61.

74. Al-Rajabi R, Patel S, Ketchum NS, et al. Comparative dosing and efficacy of sorafenib in hepatocellular cancer patients with varying liver dysfunction. J Gastrointest Oncol 2015;6:259-67.

75. Tak KY, Nam HC, Choi JY, et al. Effectiveness of sorafenib dose modifications on treatment outcome of hepatocellular carcinoma: Analysis in real-life settings. Int J Cancer 2020;147:1970-8.

76. Horger M, Lauer UM, Schraml C, et al. Early MRI response monitoring of patients with advanced hepatocellular carcinoma under treatment with the multikinase inhibitor sorafenib. BMC Cancer 2009;9:208.

77. Schraml C, Schwenzer NF, Martirosian P, et al. Diffusionweighted MRI of advanced hepatocellular carcinoma during sorafenib treatment: initial results. AJR Am J 
Roentgenol 2009;193:W301-7.

78. Shirota N, Saito K, Sugimoto K, et al. Intravoxel incoherent motion MRI as a biomarker of sorafenib treatment for advanced hepatocellular carcinoma: a pilot study. Cancer Imaging 2016;16:1.

79. Hsu CY, Shen YC, Yu CW, et al. Dynamic contrastenhanced magnetic resonance imaging biomarkers predict survival and response in hepatocellular carcinoma patients treated with sorafenib and metronomic tegafur/uracil. J Hepatol 2011;55:858-65.

80. Grillone A, Riva ER, Mondini A, et al. Active Targeting of Sorafenib: Preparation, Characterization, and In Vitro Testing of Drug-Loaded Magnetic Solid Lipid Nanoparticles. Adv Healthc Mater 2015;4:1681-90.

81. Zhang L, Gong F, Zhang F, et al. Targeted therapy for human hepatic carcinoma cells using folate-functionalized polymeric micelles loaded with superparamagnetic iron oxide and sorafenib in vitro. Int J Nanomedicine 2013;8:1517-24.

82. Lin TsT, Gao DY, Liu YC, et al. Development and characterization of sorafenib-loaded PLGA nanoparticles for the systemic treatment of liver fibrosis. J Control Release 2016;221:62-70.

83. Shi $Y$, Zhao Z, Gao Y, et al. Oral delivery of sorafenib through spontaneous formation of ionic liquid nanocomplexes. J Control Release 2020;322:602-9.

84. Thanki K, Gangwal RP, Sangamwar AT, et al. Oral delivery of anticancer drugs: challenges and opportunities. J Control Release 2013;170:15-40.

85. Gleiter H. Nanostructured materials: basic concepts and microstructure. Acta Mater 2000;48:1-29.

86. Fagin JA, Wells SA Jr. Biologic and Clinical Perspectives on Thyroid Cancer. N Engl J Med 2016;375:1054-67.

87. Kayser O, Lemke A, Hernández-Trejo N. The impact of nanobiotechnology on the development of new drug delivery systems. Curr Pharm Biotechnol 2005;6:3-5.

88. Gu M, Wang X, Toh TB, et al. Nanodiamond-Based Platform for Intracellular-Specific Delivery of Therapeutic Peptides against Hepatocellular Carcinoma. Adv Ther 2018;1:1800110.

89. Stephen ZR, Kievit FM, Zhang M. Magnetite Nanoparticles for Medical MR Imaging. Mater Today (Kidlington) 2011;14:330-8.

90. Sun T, Zhang YS, Pang B, et al. Engineered nanoparticles for drug delivery in cancer therapy. Angew Chem Int Ed Engl 2014;53:12320-64.

91. Craparo EF, Sardo C, Serio R, et al. Galactosylated polymeric carriers for liver targeting of sorafenib. Int J
Pharm 2014;466:172-80.

92. Chen J, White SB, Harris KR, et al. Poly(lactide-coglycolide) microspheres for MRI-monitored delivery of sorafenib in a rabbit VX2 model. Biomaterials 2015;61:299-306.

93. Khandare J, Haag R. Pharmaceutically used polymers: principles, structures, and applications of pharmaceutical delivery systems. Handb Exp Pharmacol 2010;(197):221-50.

94. Wu XZ, Xie GR, Chen D. Hypoxia and hepatocellular carcinoma: The therapeutic target for hepatocellular carcinoma. J Gastroenterol Hepatol 2007;22:1178-82.

95. Gaba RC, Yap FY, Martinez EM, et al. Transarterial sorafenib chemoembolization: preliminary study of technical feasibility in a rabbit model. J Vasc Interv Radiol 2013;24:744-50.

96. Ning E, Wang Z. Effect of Intrahepatic Arterial Delivery of Sorafenib on Normal Liver Tissue of Rabbit: An Experimental Study. J Adv Med Sci 2020;3:1-10.

97. Zhang L, Liu FY, Fu JX, et al. Hepatic arterial administration of sorafenib and iodized oil effectively attenuates tumor growth and intrahepatic metastasis in rabbit VX2 hepatocellular carcinoma model. Int J Clin Exp Pathol 2014;7:7775-81.

98. Li X, Yu H, Huang Y, et al. Preparation of microspheres encapsulating sorafenib and catalase and their application in rabbit VX2 liver tumor. Biomed Pharmacother 2020;129:110512.

99. Park W, Chen J, Cho S, et al. Acidic pH-Triggered Drug-Eluting Nanocomposites for Magnetic Resonance Imaging-Monitored Intra-arterial Drug Delivery to Hepatocellular Carcinoma. ACS Appl Mater Interfaces 2016;8:12711-9.

100. Cabral LKD, Tiribelli C, Sukowati CHC. Sorafenib Resistance in Hepatocellular Carcinoma: The Relevance of Genetic Heterogeneity. Cancers (Basel) 2020;12:1576.

101.Fan G, Wei X, Xu X. Is the era of sorafenib over? A review of the literature. Ther Adv Med Oncol 2020;12:1758835920927602.

102.Ren B, Wang W, Shen J, et al. Transarterial Chemoembolization (TACE) Combined with Sorafenib versus TACE Alone for Unresectable Hepatocellular Carcinoma: A Propensity Score Matching Study. J Cancer 2019;10:1189-96.

103. Kudo M, Ueshima K, Ikeda M, et al. Randomised, multicentre prospective trial of transarterial chemoembolisation (TACE) plus sorafenib as compared with TACE alone in patients with hepatocellular 
carcinoma: TACTICS trial. Gut 2020;69:1492-501.

104.Wu Y, Qi H, Cao F, et al. TACE-Sorafenib With Thermal Ablation Has Survival Benefits in Patients With Huge Unresectable Hepatocellular Carcinoma. Front Pharmacol 2020;11:1130.

105. Park JW, Kim YJ, Kim DY, et al. Sorafenib with or without concurrent transarterial chemoembolization in patients with advanced hepatocellular carcinoma: The phase III STAH trial. J Hepatol 2019;70:684-91.

106. Meyer T, Fox R, Ma YT, et al. Sorafenib in combination with transarterial chemoembolisation in patients with unresectable hepatocellular carcinoma (TACE 2): a randomised placebo-controlled, double-blind, phase 3 trial. Lancet Gastroenterol Hepatol 2017;2:565-75.

107. Qu XD, Chen CS, Wang JH, et al. The efficacy of TACE combined sorafenib in advanced stages hepatocellullar carcinoma. BMC Cancer 2012;12:263.

108. Bai W, Wang YJ, Zhao Y, et al. Sorafenib in combination with transarterial chemoembolization improves the survival of patients with unresectable hepatocellular carcinoma: a propensity score matching study. J Dig Dis 2013;14:181-90.

109.Zhu K, Chen J, Lai L, et al. Hepatocellular carcinoma with portal vein tumor thrombus: treatment with transarterial chemoembolization combined with sorafenib--a retrospective controlled study. Radiology 2014;272:284-93.

110.Aktas G, Kus T, Emin Kalender M, et al. Sorafenib with TACE improves the survival of hepatocellular carcinoma patients with more than $10 \mathrm{~cm}$ tumor: a single-center retrospective study. J BUON 2017;22:150-6.

111. Varghese J, Kedarisetty C, Venkataraman J, et al. Combination of TACE and Sorafenib Improves Outcomes in BCLC Stages B/C of Hepatocellular Carcinoma: A Single Centre Experience. Ann Hepatol 2017;16:247-54.

112. Ricke J, Klümpen HJ, Amthauer H, et al. Impact of combined selective internal radiation therapy and sorafenib on survival in advanced hepatocellular carcinoma. J Hepatol 2019;71:1164-74.

113. Chu SS, Kuo YH, Liu WS, et al. Effect of radiotherapy on survival in advanced hepatocellular carcinoma patients treated with sorafenib: a nationwide cancer-registry-based study. Sci Rep 2021;11:1614.

114.He M, Li Q, Zou R, et al. Sorafenib Plus Hepatic Arterial Infusion of Oxaliplatin, Fluorouracil, and Leucovorin vs Sorafenib Alone for Hepatocellular Carcinoma With Portal Vein Invasion: A Randomized Clinical Trial. JAMA Oncol 2019;5:953-60.

115.El-Khoueiry AB, Sangro B, Yau T, et al. Nivolumab in patients with advanced hepatocellular carcinoma (CheckMate 040): an open-label, non-comparative, phase 1/2 dose escalation and expansion trial. Lancet 2017;389:2492-502.

116.Zhu AX, Finn RS, Edeline J, et al. Pembrolizumab in patients with advanced hepatocellular carcinoma previously treated with sorafenib (KEYNOTE-224): a non-randomised, open-label phase 2 trial. Lancet Oncol 2018;19:940-52.

117. Hato T, Zhu AX, Duda DG. Rationally combining antiVEGF therapy with checkpoint inhibitors in hepatocellular carcinoma. Immunotherapy 2016;8:299-313.

118. Rivera LB, Meyronet D, Hervieu V, et al. Intratumoral myeloid cells regulate responsiveness and resistance to antiangiogenic therapy. Cell Rep 2015;11:577-91.

119. Terme M, Colussi O, Marcheteau E, et al. Modulation of immunity by antiangiogenic molecules in cancer. Clin Dev Immunol 2012;2012:492920.

120. Chen Y, Ramjiawan RR, Reiberger T, et al. CXCR4 inhibition in tumor microenvironment facilitates antiprogrammed death receptor- 1 immunotherapy in sorafenib-treated hepatocellular carcinoma in mice. Hepatology 2015;61:1591-602.

121.Zhou Z, Qin H, Weng L, et al. Clinical efficacy of DCCIK combined with sorafenib in the treatment of advanced hepatocellular carcinoma. J BUON 2019;24:615-21.

122. Ye L, Mayerle J, Ziesch A, et al. The PI3K inhibitor copanlisib synergizes with sorafenib to induce cell death in hepatocellular carcinoma. Cell Death Discov 2019;5:86.

123. Ardelt MA, Fröhlich T, Martini E, et al. Inhibition of Cyclin-Dependent Kinase 5: A Strategy to Improve Sorafenib Response in Hepatocellular Carcinoma Therapy. Hepatology 2019;69:376-93.
Cite this article as: Eresen A, Zhang Z, Yaghmai V. Strategies to improve sorafenib efficacy during image-guided treatment of hepatocellular carcinoma. Ann Transl Med 2021;9(23):1745. doi: 10.21037/atm-21-3768 